\title{
Why and how chloroquine, hydroxychloroquine and herbal antimalarials relieve most clinical manifestations of corona virus disease of 2019 (COVID-19)
}

\author{
Uchechukwu Anastasia Utoh-Nedosa* \\ Department of Pharmacology and Toxicology, Faculty of Pharmaceutical Sciences, Nnamdi Azikiwe University, Nigeria
}

\begin{abstract}
The aim of this paper is to is to explain how and why hydroxychloroquine or chloroquine works in the relief of most of the severe manifestations of covid-19. Some people have reported successful covid-19 treatment results with chloroquine or hydroxychloroquine but others have not. Chloroquine, and its derivative hydroxychloroquine; mepacrine and herbal antimalarials are used to treat both malaria and other conditions like arthritic, rheumatoid, Lupus erythematosus and pain-relief conditions. They are also used to control parasitic worm infestations. The recent use of the antimalarial drugs chloroquine and hydroxychloroquine for the management of covid-19, is thus not the first time orthodox and herbal antimalarial drugs are applied as treatment for conditions other than malaria. In this new use for the treatment of covid-19, hydroxychloroquine is used as a main drug assisted by other drugs like Azithromycin or Doxycycline and the mineral element zinc. The paper suggests that the similarities between the nature of infectivity and the manifestations of clinical malaria and those of clinical COVID-19 disease is the reason why chloroquine, hydroxyl-chloroquine and herbal antimalarial preparations "work", in those covid-19 patients in whom they worked. Chloroquine, Hydroxychloroquine, mepacrine or herbal antimalarials used in the treatment of COVID-19 work by occupying the cells of the body of the covid-19 patient and that of the virus. While "bound" to these sites, the antimalarial inhibits excessive energy utilization by the virus and the patient's own distressed body cells. The inhibition of excessive energy utilization of the corona viruses produced by the hydroxy-chloroquine or chloroquine or herbal antimalarials, inhibits the corona virus proteins from exerting excessive excitatory and oxidative stress on the cells of the tissues of organs of the COVID-19 patient. Inhibition effects produced by the hydroxychloroquine or other efficacious antimalarials also relaxes skeletal, smooth and cardiac muscles of the patient thereby, inhibiting excessive oxidation and damage of the muscles of various organs (the blood and the vital organs of the patient like the lungs, heart, liver, intestines, spleen and the kidneys), of the antimalarial treated-covid-19 patient. The inhibitory occupation of the blood and tissues of organs of the covid-19 patient, by the antimalarial exercises non-specific anti-oxidant radical scavenging activities that breakdown and mop-up blood clots or other tissue damages wrecked by the excessive excitatory activities of the corona viruses, the active haem they released from haemoglobin of attacked erythrocytes and the corona virus toxins. The administered hydroxychloroquine or other antimalarial, also stimulates the production of increased numbers of new red and white blood cell including in extra-bone marrow sites of erythropoiesis (like the spleen, the lungs and the kidneys). The newly produced erythrocytes replenish the erythrocytes damaged or incapacitated by corona viruses and so reverse silent hypoxia that existed in the internal organs of many covid-19 patients. Lastly, the administered anti-malarial also boost the immune system of the patient. The boosted immune system of the patient is then better equipped to resist the corona virus attack.
\end{abstract}

\section{Introduction}

Viruses live and multiply themselves only inside living cells. Viruses gain entrance to or infect a cell and replicate themselves after copying the genetic apparatus of the invaded cell. This mode of life of viruses makes it virtually impossible for antibiotic drugs and agents to inhibit or kill viruses because antibiotics usually inhibit or kill pathogenic organisms by inhibition or perforation of their cell walls; by inhibition of the energy-metabolism of their cells or by inhibition of their protein synthesis. Such anti-biotics do not effectively antagonise viruses because viruses do not live as independent cells and do not have cell walls. This is why chemotherapeutic agents find it difficult to eliminate pathogenic plant, animal or human viruses. The immune system of a plant, animal or human is thus very essential for the defence of that plant, animal or human, from virus infections including covid-19.

The common cold virus produces some or all of the following symptoms in patients: running nose; alternately blocked nostrils; sneezing, coughing, watery eyes; fever; catarrh; red eyes; and sometimes sore throat, to varying degrees. A patient may have a combination of some or all of these symptoms. A rare symptom of common cold is the presence of 'slabs' of thickened mucus that block the vocal cords and makes the patient to be unable to vocalise words (to talk).

The droplets from the sneeze or cough of a common cold patient who is coughing and / or sneezing can infect an un-infected person within three to six feet of the patient. Despite these numerous distressing symptoms of the attack of common cold virus, it is self-limiting. It takes four days to one week for the common cold symptoms to disappear in almost all patients, if the patient rests in bed, for at least 3 of the peak days of the manifestation of the common cold symptoms. The speed of this recovery from common cold virus attack without drug treatment is correlated to the level of immunity of the patient and is quickened by eating a lot of health-promoting diets and drinking a lot of healthpromoting fluids, including hot water.

*Correspondence to: Uchechukwu Anastasia Utoh-Nedosa, Department of Pharmacology and Toxicology, Faculty of Pharmaceutical Sciences, Nnamdi Azikiwe University, Awka, Anambra State, Nigeria, Email: aunedosa@gmail.com

Received: August 06, 2020; Accepted: August 19, 2020; Published: August 25, 2020 
In hospitals, patients showing symptoms of the attack of common cold virus are treated with fever-relieving drugs like paracetamol and cough-relieving or anti-tussive drugs if they have cough. An antibiotic is only added to the fever-relieving and cough-relieving drugs for a patient suffering from common cold virus attack, if the patient has severe and disabling sore throat infection like the one that disables talking in the patient.

This example given with the attack of common cold virus which by the way belongs to the corona group of viruses demonstrates that the most important factor in the resistance of common cold virus attack or the recovery from its distressing symptoms is the building of a formidable immune system by the patient and rest in bed. Drugs given to the patient suffering from common cold virus are not really common cold virus-killing drugs. They are drugs that help calm the distressed body tissues of the patient (paracetamol relieves the fever of the patient while anti-tussive drugs silence the cough of the patient).

Antibiotics alone cannot be relied on to inhibit and drastically reduce parasitaemia of corona viruses or to kill them. Antimalarial drugs which are technically another class of drugs, were therefore called-in like an army division requesting a regiment from a separate division of the army to help ward-off a daunting offensive facing them. This is why the treatment arsenal employed in the COVID-19 treatment front lines often include different substances like zinc (an essential dietary element), an antimalarial, hydroxychloroquine and an antibiotic azithromycin or doxycycline, assembled to both attack the corona viruses and to stabilize the body of the patient. As with drugs employed in the relief of distressing symptoms of common cold virus attack, drugs chosen for employment in the management of the distressing symptoms of covid-19 are assembled to calm the distressed body of the covid-19 patient, kill or disable the corona viruses and enable the patient overcome the attack of a virus.

Chloroquine and its hydroxyl-derivative, dihydro-chloroquine are drugs originally used for the treatment of malaria. Malaria is caused by protozoa (malaria-causing parasites), which are one-celled animals. The protozoa that cause malaria are plasmodia. The four plasmodium species which cause most malaria in the world include Plasmodium vivax, Plasmodium falciparum, Plasmodium ovale and Plasmodium malariae. Malaria remains one of the most important distressing diseases in the world. This, I think was the basis of choosing antimalarial drugs to combat covid-19 which is a very distressing disease for those who do not have high immunity.

Malaria was treated for centuries with extracts of the bark of a plant, Cinchona. The alkaloid of Cinchona, quinine was the most generally applied orthodox antimalarial used in the world till the development of alternatives like chloroquine.

Other examples of anti-malarial drugs apart from quinine are chloroquine and hydroxychloroquine; pamaquine; pyrimethamine; quinidine; mefloquine; and artemisinin-based anti-malarial drugs like artesunate and artemether, just to mention few.

Azithromycin is a macrolide antibiotic like Erythromycin, Clarithromycin and Roxithromycin. Macrolide antibiotics are generated from the soil borne bacteria Saccharopolyspora erythraea. Macrolide anti-biotics inhibit bacterial RNA-dependent protein synthesis against gram positive-bacteria like gram-positive cocci. Examples of grampositive bacteria are Staphylococci and Streptococci. Macrolide antibiotics also inhibit intracellular pathogenic organisms like Chlamydia, mycoplasma and Ligionella. The action of macrolide antibiotics is primarily bacteriostatic but at high doses, they can be bactericidal, depending on the organism they are acting on.

Hydroxychloroquine is in many instances combined with azithromycin, so we now know that hydroxychloroquine will confront corona virus and covid-19 clinical symptoms as it confronts malaria parasite, plasmodium and clinical malaria symptoms. Azithromycin will confront corona virus as a gram- + -bacterial protein-synthesis inhibitor.

I write this article on why and how chloroquine, hydroxychloroquine or herbal antimalarials employed in treatment of corona virus disease (covid19), worked in those patients who experienced relief from COVID-19 distressing symptoms, from their use, as someone who has taken chloroquine, mepacrine (quinacrine), camoquine and antimalarial herbal preparations many times; given them to members of my family, many times; seen chloroquine administered as an antimalarial in hospitals to many malaria patients and as someone who has done some animal studies with chloroquine, mepacrine and dihydroartemisinin.

I see cough as a major symptom of COVID-19 whether or not the patient has rapid breathing, difficulty in breathing or pneumonia. This is because cough is a reflex that attempts to clear the respiratory system of agents that the body deem harmful to it. Persistent cough is the body "shouting" that there are some dangerous corona viruses or toxic materials in the respiratory system of the covid-19 patient that the cough is trying to expel.

This paper discusses factors which make it possible for antimalarials like hydroxychloroquine or herbal antimalarial preparations to be effective in relieving the distressing and many times fatal clinical symptoms of covid-19, in those patients who got relieved when these antimalarials were given to them.

\section{Factors which enable the antimalarial hydroxychloro- quine to be effective in relieving the clinical signs and symptoms of COVID-19}

There are many resemblances between the incubation period of the infective organism and the signs, symptoms and clinical manifestations of malaria and those COVID-19. These resemblances are what make antimalarial drugs used to treat clinical malaria to also relieve most clinical manifestations of COVID-19. These similarities will be presented individually below.

Similarity of the incubation period of malaria parasite and corona virus: The malaria parasite plasmodium (of which four main species cause most malaria in the world: Plasmodium vivax, Plasmodium falciparum, Plasmodium ovale and Plasmodium malariae), has an incubation period of an average of 10 to 14 days which can occasionally be as low as $4-7$ days. The incubation period of corona virus is an average of 5-6 days but can be up to 14 days (Centers for Disease Control and Prevention [CDC] COVID-19 Alert April 13, 2020)

\section{Similarities between the signs and symptoms of clinical covid-19 and those of clinical malaria:}

Symptoms of covid-19: The following are the symptoms of COVID-19: Fever; cough; shortness of breath; difficulty breathing; trouble breathing; chills; repeated shaking with chills; muscle pain; persistent pain and pressure in the chest; headache; sore throat; new loss of taste or smell; new confusion; inability to arouse; bluish lips or 
face and deaths (Centres for Disease Control and Prevention [CDC] COVID-19 Alert April 13 2020).

Symptoms of clinical malaria: The symptoms of clinical malaria include malaise and an initial chill or shivering which lasts for 1560 minutes. This chill is succeeded by a spiking fever that frequently reaches $400 \mathrm{C}$ (or more than $410 \mathrm{C}$ in $\mathrm{P}$. falciparum malaria). This fever lasts several hours, subsides for less than an hour or few hours and reoccurs. This fever occurs daily, every 2 days or every three days. The length of time in between the relapses of this fever depends on which Plasmodium species caused the malaria and on the immunity of the malaria victim. This recurring nature of malaria fever makes the fever to be referred to as relapsing fever.

Summary of the similarities between the symptoms and pathophysiological changes in malaria and COVID-19 patient: The (Table 1) below summarizes the similarities between many of the symptoms and pathophysiological changes in malaria and COVID-19 patient which makes it possible for chloroquine, hydroxychloroquine or herbal antimalarial medicines to largely relieve the distressing symptoms and organ effects of COVID-19.

The similarities between many of the symptoms and pathophysiological changes in malaria and COVID-19 patients make antimalarial drugs to be able to relieve most of the distressing symptoms and signs of COVID-19 in those patients in whom these antimalarial drugs were efficacious (worked).

\section{Two-stage invasion of the human body by both malaria parasite and corona virus:}

Two-Stage Invasion of the Human Body by Malaria Parasites.

Pre-erythrocytic or hepatic stage of multiplication of malaria parasites: In malignant malaria, the malaria parasite injected into human blood by an infected mosquito leaves the peripheral blood and enters the liver where it multiplies in the liver cells. This stage of the malaria parasite cycle is called the pre-erythrocytic cycle. The multiplication of the malaria parasites inside the liver cells is called the pre-erythrocytic - schizogony.

Erythrocytic stage of multiplication of malaria parasites: When the parasites are released from the liver cells, they enter the blood and multiply inside the red blood cells. The growth and multiplication of malaria parasites inside the red blood cells of a patient is called the erythrocytic stage of the life cycle of the malarial parasite. The multiplication of the malarial parasites inside the red blood cells or erythrocytes, is called erythrocytic schizogony.

\section{Two-stage invasion of the human body by corona viruses}

Pre-erythrocytic or pulmonary stage of multiplication of corona viruses: In covid-19, corona virus gets into the respiratory tract through the nose or mouth and first invades or multiplies in the tissues of the lungs. Dr Alan pointed out that chest X-rays and CT scans of even covid-19 patients who have no or few respiratory complaints show radiological signs of pneumonia [1], which is an evidence of multiplication of corona viruses in the lungs and destruction of lung parenchyma cells of the covid-19 patient. There is evidence of damage to the lungs and formation of immune complexes by corona viruses in the lungs. Dr Duc Vuong, a medical doctor and a surgeon, in a video presentation in YouTube which he called "how COVID-19 kills", described and demonstrated the mechanisms of the colliquative pneumonia and distressed-breathing stage of the attack of COVID-19 [2].

Erythrocytic Stage of Multiplication of Corona Viruses: Corona viruses released from lung tissues enter the blood where they seem to multiply in and damage red blood cells. This is evidenced by the fact that, as Dr Alan pointed out, covid -19 patents "are experiencing blood clotting and low oxygen levels" [1] which suggest that corona virus attack of the red blood cells has damaged the red blood cells and destroyed the haemoglobin of the erythrocytes to cause low oxygen levels.

The fate of the red blood cells at the end of the erythrocytic multiplication of malaria parasites and corona viruses

At the end of the erythrocytic cycle, the malaria parasites or corona viruses have damaged the globin, the protein portion of haemoglobin and released the haem of haemoglobin. The infected red blood cell or the corona virus-infected erythrocytes lyse releasing malaria parasite toxins or corona virus toxins (which are antigenic) and other residues.

\section{The clinical stage of malaria attack}

Clinical malaria occurs in the signet ring stage of the erythrocytic schizogony when, a large vacuole displaces the nucleus of the cell of the schizont to one side. When this vacuole disappears, haemozoin pigment granules, are deposited. The nucleus of the schizont then divides several times and cytoplasm surrounds each nucleus to form large numbers of new small malaria parasites called merozoites. The infected red blood cells or erythrocytes eventually rapture releasing the merozoites; haemozoin and other metabolic wastes, at definite synchronized intervals characteristic of the malaria of different Plasmodium species. These intervals constitute the periods during which the affected person displays signs and symptoms of malaria. Some merozoites develop into

Table 1. A comparison of the signs, symptoms and pathophysiological changes of malaria and COVID-19 which highlights the similarities that exist between them Signs and Symptoms and Pathological changes Observed in COVID-19

Malaise (feeling unwell); inability to arouse; sore throat; Muscle pain; Headache; loss of taste and smell

Cough or No cough in some patients.

(Repeating) Fever which can reach $102-104^{\circ} \mathrm{F}$

Chills

Repeated shaking with chills

Distressed breathing; Rapid, difficult shallow breathing or shortness of breath, pneumonia

Blood pathological changes; Bluish lips or face; Internal bleeding and internal formation of blood clots.

Signs and Symptoms and Pathological changes Observed in Malaria

Signs of diminished function of vital organs like the lungs, liver and kidney.

Malaise (feeling unwell); general debility; Lack of appetite; Loss of sense of taste

Repeating or Relapsing Fever which can reach $102-104^{\circ} \mathrm{F}$

Chills

Repeated Shivering and Intermittent rigors

Diminished blood flow; Anaemia; whitish pale palms and eyeballs showing diminished blood supply to those areas; body weakness; lack of strength

Signs of diminished function of vital organs like the lungs, liver and kidney like centrilobular congestion and degeneration in the liver; bitter taste in the mouth; yellow coloured lips, palms, and cornea of the eye.

Oligourea and the presence of casts, protein and red cells in the urine ${ }^{5}$ 
gametocytes within the erythrocytes. The merozoites released into the blood can go back and re-infect the liver or infect new red blood cells.

\section{The clinical stage of covid-19 attack}

The peak of the clinical stage of covid-19 attack is often marked by a combination of many of these signs and symptoms: high fever that may reach $102-104^{\circ} \mathrm{F}$, cough; shortness of breath; difficulty breathing; trouble breathing; chills; repeated shaking with chills; muscle pain; persistent pain and pressure in the chest; headache; sore throat; new loss of taste or smell; new confusion; inability to arouse; bluish lips or face. Repeated shaking with chills and intermittent fever in a covid-19 patient indicate that corona virus-infected erythrocytes burst intermittently to release viral toxins and active haem which can cause oxidative damage to tissues of the covid-19 patient, Each new release of the newly multiplied parasites and toxins released with them from the lysed infected red blood cells, poisons the cells of the body causing the generation of the chills and shivering. Severe symptoms may result in deaths in some patients. There is also presence of blood clots in autopsies of some patients.

\section{The fate of haem released from damaged haemoglobin of erythrocytes in malaria}

Blood parasites like the malaria parasite (Plasmodium species), Rhodnius spp and Schistosoma spp, are able to digest haem released from haemoglobin when the parasite fed on the contents of the red blood cell during its growth phase (before it replicated itself into numerous new parasites). Haem is toxic to cells, but these blood parasites digest it to haemozoin which is the insoluble crystalline form of haem.

\section{The fate of haem released from damaged haemoglobin of erythrocytes in COVID-19}

Since corona virus is not a blood parasite, it is likely that when the corona virus-infected erythrocyte bursts, it releases toxic haem, corona virus toxins, new brood of corona viruses and toxic wastes of corona viruses, into the blood of the covid-19 patient.

\section{Local pathological changes which result from clinical malaria}

Many local pathological changes occur as a result of clinical malaria. They include the following:

Intermittent rigors or chills and anaemia with enlargement of the spleen: Pathologic multiplication of a Plasmodium species (the development of malaria parasitaemia) in humans, produces intermittent rigors or chills and anaemia with enlargement of the spleen.

Permeability of the capillary walls, slowing and stoppage of the blood flow; concentration of the red blood cells; and reduction and redistribution of the renal circulation: Clinical malaria results in permeability of the capillary walls resulting in escape of fluid from the blood into the tissues; slowing and stoppage of the blood flow; concentration of the red blood cells; and reduction and redistribution of the renal circulation.

Presence of detectable varying degrees of anaemia: During the paroxysms of clinical malaria, normocytic anaemia of variable severity may be detected: with transient leucocytosis and subsequent leucopenia with a relative increase in large mononuclear cells.

Low oxygen level in the liver: Local pathological changes which result from malaria also result in a reduction of the amount of oxygen available to the liver parenchyma cells; centri-lobular congestion and degeneration in the liver. The oxygen requirements of the parasites themselves aggravate the oxygen needs of the host tissues.

Effect on liver bilirubin and liver function enzymes: Due to local pathological changes which result from malaria, total bilirubin concentration is often significantly raised with the unconjugated component being higher. Thus, malaria may cause liver damage resulting in jaundice due to hyperbilirubinemia. There is often also, significant elevation of alkaline phosphatase activity and increase in transaminase levels in malaria. There was an association between malaria in pregnant women and the levels of serum glutamate oxaloacetate transaminase (SGOT), serum glutamate pyruvate transaminase (SGPT) and serum alkaline phosphatase (ALP) activity of the blood. Liver function tests may also give abnormal results during malaria attacks.

Neurologic pathological changes due to malaria: When congestion and degeneration occur in the brain capillaries as a result of local pathological changes due to malaria, brain complications result and may give rise to cases of coma ending fatally in untreated first infections with Plasmodium falciparum.

Effect of malaria attack on the kidney: In severe $P$. falciparum malaria infections, renal damage may cause oligourea and the presence of casts, protein and red cells in the urine.

\section{Local pathological changes that occur in the blood and blood vessels of COVID-19 patients}

Local pathological changes that occur in the blood and blood vessels of covid-19 patients are manifested as bluish lips or bluish face and presence of blood clots in autopsies of some patients.

Many doctors report that they are seeing blood clots or gel-like clumps in the blood of an alarming number of covid-19 patients. Dr Jeffrey Lawrence, a haematologist at Weill Cornel Medicine, in New York city told CNN, "the number of blood clotting problems I am seeing in the intensive care unit in covid-19 patients, is unprecedented"[3]. Some hospital workers reported they were having trouble with dialysis machines used for covid-19 patient who have blood clots, as clots in the blood of such patients clogged the machine tubing.

A recent study on covid-19 patients in Netherlands published in the Journal of Thrombosis Research found that 30\% of 184 covid-19 patients had some clotting issues [3].

There were also reports that some relatively young people in their 30 s and 40s confirmed to be infected with coronaviruses, were having strokes [3]. Because of the potential danger and possible lethality associated with these blood clots in covid-19 patients, some hospitals put covid-19 patients on low doses of blood thinners to prevent clots [3]. Despite this approach, some doctors noticed that their covid-19 patients were developing clots in their legs even while they were on blood thinners [3].

\section{COVID-19 lowered oxygen level in the blood of covid-19 patients and silent hypoxia}

Dr Ron Elfenbein, an Emergency Care physician, speaking on the topic of "Silent hypoxia" (also called "happy hypoxia") in corona virus patients, pointed out that silent hypoxia is seen in some corona virus patients in emergency care who show a low oxygen-saturation level of 50 t0 $70 \%$ [when normal individuals have an oxygen saturation level of ninety nine percent (99\%)] [4]. Oxygen saturation level measures 
the percentage of an individual's red blood cells that have oxygen. Some people have suggested that there may be a "hypocoagulative" state in the silent hypoxia condition of covid-19 patients [4]. The low oxygen saturation level observed in COVID-19 patients suggests that corona virus attack damages the haemoglobin of the red blood cells. Haemoglobin functionally picks up oxygen from the alveoli of the lungs and transports it to the tissues of the body where it collects carbon dioxide produced as waste by the tissues of the body to discharge them at the alveoli of the lungs. From the alveoli of the lungs, carbon dioxide exits the body during expiration.

Haem group of haemoglobin contains iron $(\mathrm{Fe})$ at the centre of the group. The iron in haem is in ionic state and acts by changing from ferric $(\mathrm{Fe} 3+)$ to ferrous $(\mathrm{Fe} 2+)$, state, and vice versa as it picks up or drops oxygen to the tissues of the body. As the charged iron radical changes from its ferric to ferrous state, it picks up oxygen or carbon dioxide by an oxidation- reduction reaction.

Corona viruses that attack red blood cells attach themselves to and destroy the protein group of haemoglobin, globin, and displace the haem group of haemoglobin which is the actual transporter of oxygen and carbon dioxide in the body. This displacement and discarding of the haem of the haemoglobin of invaded erythrocytes is what causes many red blood cells of the covid-19 patient to be devoid of oxygen resulting in low oxygen saturation level of the patient's red blood cells.

Lysed virus-attacked erythrocytes release active haem and viral toxins which are antigenic into the blood of covid-19 patients, to cause non-specific radical oxidative damage to the blood and internal organs of the patient

When erythrocytes attacked by erythrocytic corona viruses lyse, the haem group of haemoglobin which they release into the blood together with the corona virus toxins amount to release of ionic iron (which are charged particles capable of causing severe oxidative damage), into the blood to damage cells of the blood and cells of tissues of internal organs of the body of affected covid-19 patients. It is likely that oxidative damage produced by this haem and viral toxins, causes the gel-like clumps of blood seen in many COVID-19 patients as well as cellular damage that deteriorate the functioning state of internal organs of covid-19 patients, like the liver and the kidney. For example, the reports that some relatively young people in their 30 s and 40 s confirmed to be infected with coronaviruses, were having strokes [5], suggests that covid-19 attack damages brain vascular tissues.

\section{Covid-19 attack incapacitates skeletal muscles of the covid-19 patient}

The covid-19 symptoms of muscle pain, shortness of breath; difficulty breathing; trouble breathing; persistent pain and pressure in the chest; and general debility, all suggest that corona virus attack exerts excessive oxidative stress and excessive cellular excitatory pressure on the diaphragm and the internal and external intercoastal muscles that control breathing-in (inhalation) and breathing-out (exhalation). General debility and poor ability to move about by the covid-19 patient is an indication of the presence of oxidative stress in the ambulatory muscles of the covid-19 patient.

\section{There is clear evidence that COVID-19 destroys the tissues of the lungs of covid-19 patients}

Colliquative necrotic pneumonia in which the covid-19 patient breathes as if he is breathing through water or is drowning in the liquid in his lungs [6], and sore throat suffered by many COVID-19 patients as clinical manifestations of severe COVID-19, are clear indications that corona viruses damage and destroy the lung parenchyma cells and cells of smooth muscles that line the respiratory tract.

\section{Covid-19 destroys the tissues of the internal organs of the covid-19 patients}

Evidence that the effects of covid-19 destroy many vital organs of the patient or brings down their level of functioning was pointed out by Dr Allan Detsky, a professor in the institute of health policy, management and evaluation and in the department of medicine of the University of Toronto [7]. He noted that in addition to destroying the lungs of covid-19 patients, covid-19 also diminishes the function of many internal organs of covid-19 patients because many patients have been noted to experience damage to organs far away from the lungs, that include the kidneys, heart, brain and blood clotting systems" [7].

\section{Covid-19 adversely affects the tissues of the sense organs and the nervous system of some covid-19 patients}

The covid-19 symptoms of 'new loss of taste or smell'; "new confusion" and "inability to arouse", noted in some Covid-19 patients, suggest that COVID-19 attack exerts a disorientating and possibly damaging effect on the sense organs, and the peripheral and central nervous system of some COVID-19 patients.

Discussions on how and why Scizonticidal Antimalarials Largely Relieve the Manifestations of Clinical COVID-19:

How schizonticidal antimalarials like chloroquine, dihydroartemisinin, hydroxychloroquine and schizonticidal herbal anti-malarials, work in the treatment of COVID-19

Antimalarial drugs are used and widely advertised for their malaria parasite schizonticidal actions. Chloroquine will be used here as a representative drug. Chloroquine is a schizonticidal and gametocidal antimalarial drug. Chloroquine has a $90 \%$ bioavailability and concentrates in melanin- containing cells in the skin, eyes. kidneys, liver and lungs.

These properties of chloroquine indicate that when chloroquine or hydroxychloroquine is administered to a COVID-19 patient, in appropriate doses, the chloroquine or hydroxychloroquine will exert "schizonticidal and gametocidal" action on corona viruses that will kill them or at least give them a formidable combat. Chloroquine or hydroxychloroquine administered to a covid-patient will have a $90 \%$ bioavailability in the body of the patient and will concentrate in melanin- containing cells in the skin, the eyes, the kidneys, the liver and the lungs of the covid-19 patient. This is in fact what chloroquine or hydroxychloroquine administered to a COVID-19 patient does because they are schizonticidal antimalarials and schizonticidal antimalarials are antimalarial endoperoxides [8] and they react with specific parasite proteins and alkylate [9] such parasite proteins.

This means that chloroquine or hydroxyl-chloroquine accumulates in the corona virus-infected lung parenchyma cells or corona virusinfected red blood cells of the COVID-19 patient and kill the corona viruses inside these infected lung cells or red blood cells, (which are displacing iron $\left(\mathrm{Fe}^{-}\right)$in haemoglobin of red blood cells or bursting the red blood cells to release toxic haem and viral toxins). Similarly, the administered chloroquine or hydroxychloroquine also "concentrates in the cells of the skin, eyes, kidneys, liver and lungs et cetera, tissues 
of the COVID-19 patients to exert inhibitory anti-oxidant and antiexcitatory- protective effects.

\section{Mechanism of action of schizonticidal antimalarials like chloroquine, dihydroartemisinin, hydroxychloro- quine and schizonticidal herbal anti-malarials, in the treatment of COVID-19}

The first and most dramatic body remedying and stabilizing effect of administered hydroxychloroquine on those in whom it worked is its anti-pyretic or fever-relieving effect on the covid-19 patient. I know a covid-19 patient in whom hydroxychloroquine reduced his temperature from $104^{0 \mathrm{~F}}$ to $102^{\mathrm{OF}}$, then from $102^{0 \mathrm{~F}}$ to $98.8^{0 \mathrm{~F}}$ and lastly from $98.8^{\mathrm{OF}}$ to $94.4^{\mathrm{oF}}$, in a few hours.

This antagonism of rise of the temperature of the body to many degrees above the normal temperature of the body, by administered hydroxychloroquine, is an inhibition of excessive stimulation and derangement of the body by dangerously raised temperatures in body tissues. Anti-pyretic effect is a main effect of schizonticidal antimalarial drugs. In earlier decades like in the 1960s, the schizonticidal antimalarial quinine was commonly given to mothers in hospitals for the lowering of fevers in babies and children. So, fever-relief is actually the primary property of anti-malarial drugs, including hydroxychloroquine. Antimalarial drugs like chloroquine and hydroxychloroquine produce practically all their effects in the body the inhibiting body damage just as they prevent damage of the body (especially damage of brain cells) by excessively raised temperatures in the body (high fever). These antimalarial drugs similarly inhibit excessive muscle contractions in tissues of the body.

The results of experiments I did with chloroquine and mepacrine on isolated smooth muscles of the rectum of 1 day -1 month old chicks, showed that Chloroquine, $(1 \mu \mathrm{g}$ and $10 \mu \mathrm{g})$, produced dose-dependent antagonism (inhibition), of the biphasic tonal and electrical field stimulation responses of these smooth muscles, and produced a dosedependent antagonism of the responses of these smooth muscles to 10ng ATP [adenosine triphosphate], (unpublished findings). The assay of mepacrine on the same isolated smooth muscles of the rectum of one day to one- month-old chicks, showed that a dose of $2 \mu \mathrm{g}$ mepacrine, produced a relaxation response of these smooth muscles which lasted close to three hours when the experiment was stopped and also antagonised contraction of these muscles in response to electrical field stimulation, (Unpublished findings).

These results obtained on the assay of chloroquine and mepacrine on chick rectum smooth muscles, suggest that the common knowledge we already have in pharmacologic literature that chloroquine " concentrates in melanin- containing cells in the skin, eyes. kidneys, liver and lungs", really means that chloroquine has an "inhibitory occupation" mechanism of action. Artemisinins also said to "localise in malaria parasite infected red blood cells" [10]. This is also equivalent to saying that artemisinin has an "inhibitory occupation" mechanism of action. This inhibitory occupation mechanism of action is unknowingly acknowledged for many drugs by saying that the drug has "high protein-binding or high tissue-binding".

The dose-dependent antagonism of the responses of chick rectum smooth muscles to applied adenosine triphosphate (ATP), by chloroquine mentioned above, means that inhibitory occupation of the smooth muscles of chick rectum by chloroquine, enabled chloroquine to inhibit excessive excitation of these smooth muscles by administered adenosine triphosphate (ATP). This is the secret of the mechanism of inhibitory actions of chloroquine, artemisinin or other schizonticidal antimalarial drugs.

Inhibitory occupation of living tissues enables a schizonticidal antimalarial drug to inhibit excessive energy utilization by the drugoccupied cell and inhibits excessive oxidative phosphorylation of the occupied cells and tissues of the anti-malarial drug-treated patient. Inhibitory occupation of malarial parasite proteins or corona virus proteins by schizonticidal antimalarial drugs like chloroquine, hydroxychloroquine, mefloquine et cetera or herbal antimalarials, inhibits excessive excitatory activities and excessive oxidative stress which the malaria parasite or corona virus inflicts on the blood and tissues of organs of the malaria or COVID-19 patient. Such inhibition of excessive oxidative phosphorylation in the blood, and organs of a patient is an anti-oxidant non-specific radical scavenging activity of these antimalarial drugs.

\section{Parasite or virus-schizonticidal action}

Schizonticidal antimalarials like chloroquine, hydroxychloroquine, mefloquine and artemisinin,et cetera, accumulate in malaria parasiteinfected erythrocytes to attack and kill the malaria parasites and protect the infected erythrocytes. Schizonticidal antimalarials enter and accumulate in infected erythrocytes [8]. A study demonstrated the uptake of $\left[{ }^{3} \mathrm{H}\right]$ - dihydroartemisinin, (labelled-dihydroartemisinin), by erythrocytes infected by Plasmodium falciparum, in vitro [7]. Another study demonstrated haemolysis of Plasmodium falciparum trophozoite-infected erythrocytes after artemisinin exposure [11]. This is how schizonticidal authodox and herbal antimalarial drugs like chloroquine, hydroxychloroquine; artemisinin et cetera, accumulate in the corona virus-infected erythrocytes of covid-19 patients to kill the corona viruses and protect the infected red blood cells.

Schizonticidal antimalarials will be able to kill corona viruses because they are endoperoxides [12]; they interact with and alkylate ${ }^{35}$ specific parasite proteins [12]. A study demonstrated that schizonticidal antimalarials with alkylating properties alkylate parasite proteins best when activated by a reduced haem model ${ }^{36}$. Another study showed that Plasmodium falciparum trophozoite-infected erythrocytes haemolysed after exposure to artemisinin [11]. This shows that once the schizonticidal antimalarial drug is in the same medium with parasiteinfected erythrocytes, such infected erythrocytes will lyse. Once the infected erythrocytes lyse, they will release haem which then stimulates the ani-malarial into attachment to malaria parasite- or corona virusproteins to alkylate them.

So, the presence of haem released from corona virus- damaged red blood cells, activates hydroxychloroqine, chloroquine or other schizonticidal antimalarials to alkylate corona virus proteins, while at the same time protecting the erythrocytes and other tissues of the covid-19 patient. These schizonticidal antimalarial drugs will be able to carry out the killing of corona viruses to varying degrees, according to the level of efficacy of each different shizonticidal antimalarial for this action and according to the potency of each antimalarial administered.

\section{The inhibitory mechanism of action noted in some oth- er clinically used antimalarial drugs}

The inhibitory mechanism of action has been noted in some other antimalarial drugs. For example, the antimalarial drugs quinidine, mefloquine and halofantrine have been shown to prolong the QTinterval of the electrocardiogram (ECG). Ability to prolong the QT- 
interval of the electrocardiogram is called having a quinidine-like effect on the heart. Mefloquine is related to chloroquine and so chloroquine and hydroxychloroquine will also produce a quinidine-like effect on the heart. The anti-malarial drug, pyrimethamine (structurally related to trimethoprim) is a dihydrofoliate reductase inhibitor. Another antimalarial, proguanil is a dihydrofoliate inhibitor active against intrahepatic forms of the malaria parasite Plasmodium falciparum.

These examples show us that anti-malarials knowingly or unknowingly are employed in clinical practice for their inhibitory mechanism of action which can oppose or antagonise the devastation of body tissues by excessive excitatory activities of disease infective agents or health disorders.

Mechanism of action of azithromycin: Azithromycin acts by inhibiting protein synthesis in susceptible bacteria by binding to the 'P'-site of 50S unit of the ribosome (with which the proteins are synthesized), to inhibit RNA-dependent protein synthesis of the bacteria. Obviously, in its uses in treatment of covid-19, Azithromycin is expected to 1, Inhibit the growth of and, if possible, kill pathogenic gram-positive bacteria attacking any organs of the covid-19 patient, and 2. Inhibition protein synthesis in the corona viruses themselves.

\section{Two evidences of possible beneficial effects which ad- ministered antimalarials can give COVID-19 Patients}

Evidences of the effects of dihydroartemisinin and extracts of the herbal antimalarial plant Phylantus, highlighted below mention possible beneficial effects administered schizonticidal antimalarial drugs or schizonticidal antimalarial herbal extracts can confer to a covid-19 patient treated with schizonticidal antimalarials.

First evidence: effects of oral dihydroartemisinin: Oral doses of the antimalarial dihydro-artemisinin, produced the following effects in rats: widening of the respiratory tract [10]; erythropoietin-like effects [13]; direct stimulatory effects on the red and white blood stem cells in the Lungs [14]; dilation of hepatic blood vessels [15]; widening of the calibre of coronary blood vessels [16]; non-interference with the normal activity of liver function enzymes, alanine aminotransferase; aspartate aminotransferase and alkaline phosphatase (ALT,AST and ALP) [17]; stimulation of proliferation of intestinal smooth muscles [3]; stimulation of proliferation of lung smooth muscles [18]; stimulation of increased leucocyte production [19]; stimulation of extra-bone marrowsites of haemopoesis [20]; ability to produce self-negative feed-back [21] and immune system and body defense enhancement effects [22].

Second evidence: effects of the extracts of the herbal antimalarial plant Phylantus species: Animal and human studies done with extracts of the herbal antimalarial plant, Phylantus species, have demonstrated the following effects: HIV-1 reverse transcriptase activity [23]; antihepatotoxic activity [24]; inhibition of calcium oxalate endocytosis by renal tubular cells [25]; inhibition of calcium oxalate crystallization associated with kidney stone formation [26]; hypoglycaemic activity $[18,27]$; increasing of life span of rats with liver cell carcinoma [28]; efficacy in treating patients with chronic hepatitis ' $\mathrm{B}$ '[29]; antinociceptive (pain-relieving) property [30]; diuretic and hypotensive property [31] and inhibition of angiotensin-converting enzyme [32].

\section{Top concerns in the acceptability of an antimalarial for treatment of COVID-19}

What should be top concerns in the acceptability of an antimalarial for the management of covid-19 are:
1. The tolerability of the serious adverse side effects of the antimalarial by a patient;

2. The tolerability by the patient of the serious adverse side effects of the other drug or drugs to be combined with the antimalarial for the treatment;

3. Whether or not another drug to be combined with the antimalarial for the treatment has similar mechanism of action as the antimalarial and as such will produce additive main effects and additive adverse side effects with the antimalarial;

4. The nature of the drugs the patient had been taking prior to treatment and possible drug interactions that could occur between the antimalarial and such remnant old medications that might be in the tissues of the patient;

5. The level of immunity of the patient at treatment entry level; and,

6. The level of functioning of the vital organs such as the heart, lungs, spleen, liver, blood/blood vessels; the kidneys and the intestines of the patient at entry level for treatment.

The doctor administering the hydroxychloroquine, chloroquine or other antimalarial to a covid-19 patient must be aware of the six concerns raised above, juggle and resolve them in his or her mind before he/she administers the curative dose or otherwise of the hydroxychloroquine to the patient. Some of these factors may require the doctor to adjust the dosage or administration parameters of the antimalarial or of the other drugs combined with the antimalarial. For example, in my locality, many people are given vitamin B-complex as pre-medication to minimize the itching (which is very rampant in patients in my community), produced in patients by chloroquine and other adverse effects if chloroquine, in hospital administration of chloroquine to patients.

Those administering schizonticidal antimalarials like hydroxychloroquine to covid-19 patients, for example should know that almost all these antimalarials have many other curative properties which are not usually mentioned in the literature like diuretic property; anti-inflammatory property; quinidine-like or anti-fibrillation activity on the heart; anti-hypertensive property; anti-biotic property; anti-carcinoma property; lipolytic property and anti-hyperglycaemic property. The antimalarial drugs most commonly used in arthritic pain relief are chloroquine, hydroxychloroquine and quinacrine or mepacrine and hydroxy-chloroquine is preferred in the treatment of Lupus erythematosus. So, one cannot say from these uses of these antimalarials that it is not known in some quarters that these antimalarials are very powerful anti-inflammatory and excess fat-eliminating (lipolytic) drugs. Yet, because these body-protecting properties of these drugs are not mentioned or emphasised in medical and pharmacologic literature, someone administering these schizonticidal antimalarials to an obese covid-19 patient or a covid-19 patient with arthritic or rheumatoid conditions may not realise that when s/he administers what he thinks is the required dose for the "cure" of the covid-19, the rheumatoid conditions or heaps of fat in the obese patient takes up a lot of the curative actions of the administered drug.

Drugs do exactly what God endowed them to do when they enter the body. They exercise their remedying effects on all the defects in the body they are endowed to be able to remedy. They produce their maximal effects on all the defects they can remedy for as long as their duration of action lasts. So, the hydroxychloroquine, given to a covid-19 patient will exercise diuretic property; anti-inflammatory property; quinidine- 
like or anti-fibrillation activity on the heart; anti-hypertensive property; anti-biotic property; anti-cancer property; lipolytic property and antihyperglycaemic property, as well as anti-pyretic and other properties required to remedy the body of the covid-19 patient.

Anti-hyperglycaemic property of schizonticidal anti-malarials was noticed in quinine since 1925 [33]. This hypoglycaemic property of quinine, is referred to as an undesired adverse side effect of quinine in its use for treating malaria. So, what do you think will happen if quinine is used to treat a patient with sever malaria that is hyperglycaemic as a result of diabetes? Don't you think that the administered quinine will enter the body of the patient, and say, "well I will just relieve malaria symptoms because that is what I came to do? Of course, not, the quinine will immediately get down to work to both lower the excess blood sugar of the patient and relieve the malaria symptoms of the patient?

What relieving both serious underlying health conditions and malaria means for administered quinine is that the dosage or dosage regimen of the malaria patient that has diabetes ought to be adjusted, to enable the administered antimalarial relieve both conditions and to get good treatment outcomes [34]. The same lines of argument go for the use of schizonticidal antimalarials in treating covid-19 patients with other serious underling disease conditions or health disorders [35]. Failure to make dosage or dosage regimen adjustments to accommodate serious underlying health conditions of patients (including low immunity at treatment entry level), may contribute to poor treatment outcomes of treatments of some covid-19 patients with hydroxychloroquine. If another drug the patient is using to treat the underlying health condition is admitted to be combined with the antimalarial, then the person giving the treatment must deal with possibility of drug-drug interactions of this admitted drug with the anti-malarial being used for treatment [36].

Another property of scizonticidal antimalarials that is not known (which I observed from frequent personal use of schizonticidal antimalarials), is that these antimalarials stay bound to the site of the body that is most heavily infected with a disease or is most damaged with a health disorder, for long hours after their official biologic halflife has been completed in the body [37]. For example, if the normal duration of action of a fungicidal antimalarial is 6 hours, that fungicidal antimalarial will stay bound to, say, the ankles of the feet of a patient for another 12 hours to 18 hours, long after its normal duration of action of six has passed; if the ankle area of that patient harbours the highest concentration of the fungal infection being treated with the antimalarial. During all these 6 plus 12 or 18 hours, the patient will feel heat and stinging sensations at this ankle site as the antimalarial stings and peroxidises the fungal hyphae attached to the tissues of this ankle site [38]. Heat is released as a product of the peroxidation activities of the antimalarial and this heat can be felt by both the patient and an outsider that places the palms of his/her hands at this ankle site of peroxidation of the fungal infections. This information is important in the use of schizonticidal antimalarials like hydroxychloroquine, for the treatment of covid-19 patients, some of which may also have sites of heavy concentrations of infections or heavily disordered parts of body organs, afflicted with health disorders.

Adverse effects of chloroquine: Adverse effects of chloroquine usually include skin rashes and itching; gastrointestinal effects; headaches; vertigo; blurring of vision; ototoxicity; blood dyscrasias; cardiovascular effects; dizziness; nausea; hypotension; convulsions and cardiovascular collapse.

Adverse effects of azithromycin: The macrolide antibiotic Azithromycin has the following side effects:
Common side effects: Nausea; vomiting; diarrhoea and abdominal pain.

Other side effects: Fever; Flu-like symptoms; skin rashes; yellowing of skin; yellowing of the eyes; swollen glands; bruising; muscle aches and severe weakness.

Rare side effects: Life-threatening allergic reactions; clostridium difficile-associated diarrhoea; irregular heart- beats and liver damage.

\section{Conclusion}

The best way to resist corona virus infection or to reduce the severity of the manifestations of covid-19 is to have a strong immune system by eat healthy diets that boost the immune system. A person with a high immunity is less susceptible to any disease infection (including corona virus infection), than another person with low immunity.

Administration of appropriate curative doses of schizonticidal antimalarial drugs like hydroxychloroquine, chloroquine, mefloquine, dihydroartemisinin or schizonticidal herbal antimalarial medicinal preparations to a COVID-19 will immediately stop the fever of covid-19 patient [whose temperature can go up to $102^{\mathrm{OF}}$ to $104^{\circ},\left(40^{\circ} \mathrm{C}\right.$ to $\left.41^{\circ} \mathrm{C}\right)$ ], within a few minutes to a few hours.

In the same way as the administered antimalarial stopped the fever of the covid-19 patient it will systematically antagonise the devastating effects of covid-19 disease on the blood, the blood vessels, the muscles and the tissues of various vital organs and nervous system of the patient, through its inhibitory occupation and anti-oxidant mechanisms of action. The administered antimalarial will also, assist the body's immune system to kill the corona viruses; will stimulate new hemopoiesis to replace red and white blood cells destroyed by the virus attack; relieve hypoxic state in which the tissues of the vital organs of the covid-19 patient are functioning and boost the patient's immune system. A boosted immune system helps the recovering covid-19 patient to ward-off any remnant attack of the covid-19 disease. These viruskilling and organ-protective effects of the administered antimalarial, will enable most antimalarial-treated covid-19 patients to recover from the covid-19 attack.

\section{References}

1. Tailor W, Robert J, Nicholas J (2004) Antimalarial Drug Toxicity: A review. 27: 25-61

2. Duc Vuong, www.youtube.com https//youtu.be/4JOd59dd-qM

3. Utoh-Nedosa UA (2011). Dihydroartemisinin-Stimulated Proliferation of Rat Intestinal Smooth Muscles. Am J Appl Sci 9: 177-179.

4. Dr Duc Vuong, How COVID-19 Kills_-I'm a Surgeon—And Why We Can't Save You Dr Duc Wuong, World's No 1 Weight Lo. (Available at: https//youtu.be/4JOd59dd-qM)

5. Dr Ron Elfenbein (2020) State of "Happy Hypoxia" or "Silent Hypoxia" in Corona Virus Patients: what it Means, CBSN. (Available at: https://youtube/UJcfSbE291A)

6. Asawamahaskda W, Ittratt I, YM Pu, Ziffer H, Meshnick SR (1994). Reaction of antimalarial endoperoxides with Specific Parasite Proteins. Antimicrob Agents Chemother 38:1854-1858 [Crossref]

7. Detsky AS, Bogoch I (2020) It is not hopeless: These doctors explain what COVID-19 has in common with diseases we know how to manage-Forbes

8. Robert A, Meunier B (1998) Alkylating properties of antimalarial artemisinin derivatives and synthetic trioxanes when activated by a reduced haem model. Chem Eur J 4:1287-1296.

9. Orji AU (1996) Haemolysis of Plasmodium falciparum trophozoite-infected erythrocytes after artemisinin exposure. Br J Haematol 92: 324-328.

10. Uchechukwu UN, Goddy N, Kenechi N, Peter AA, Theophilus O, eta 1. (2011) Respiratory Tract-Widening Effects of Dihydroartemisinin in Wistar Albino Rats. Am J Pharmacol Toxicol 6: 46-48. 
11. Gu HM, Warhurst DC, Peters W (1984) Uptake of [3H] - dihydroartemisinin by erythrocytes infected by Plasmodium falciparum in vitro. Trans $R$ Soc Trp Med Hyg 78: $265-270$.

12. Ellis DS, Li ZL, Cu HM, Peters W, Robinson BL, et al. (1985) The Chemotherapy of rodent malaria, XXX1X Ultra structural changes following treatment with artemisinin of Plasmodium bergei infection in mice, with observations of the localization of [3H] -dihydroartemisinin in P. falciparum in vitro. Ann Trop Med Parasitol 79: 367-374

13. Utoh-Nedosa UA (2011) Erythropoietin-like Effects of Dihydroartemisinin in Wistar Albino Rats. Am J Agric Biol Sci 6: 511-516.

14. Utoh-Nedosa UA, Akah PA, Nedosa KS, Onyedibe IK, Nedosa IV, et al. (2011) Direct Stimulatory Effects of Oral Dihydroartemisinin on the Red and White Cell Stem Cells in the Lungs of Rats. Mol Pharm 4.

15. Utoh-Nedosa UA, Achunike AP, Stanislaus NK, Kenneth OI, Valentine NI, et al, (2012) Dilation of Hepatic Blood Vessels by Oral Dihydroartemisinin in Wistar Albino rats. Inventi Rapid: Mol Pharm 1.

16. Utoh-Nedosa UA, Akah PA, Nedosa KS, Onyedibe IK, Nedosa IV, et al. (2011) Dihydroartemisinin-Stimulated Widening of the Caliber of Blood Vessels of the Coronary Circulation in Rats. Mol Pharm 4.

17. Utoh-Nedosa UA, Mgbojikwe L, Ojegbu FN, Hawa B, Abubakar A, et al. (2011) The Effects of Oral Dihydroartemisinin on the Enzyme Activities of Serum Alanine Aminotransferase, Serum Aspartate, Aminotransferase and Alkaline Phosphotase Enzymes of Wistar Albino Rats. Mol Pharm 3.

18. Rajeshkumar NV, Kuttan R (2000) Phylantus amarus extract administration increases the lifespan of rats with hepatocellular carcinoma. J Ethnopharmacol 73: 215-219 [Crossref]

19. Utoh-Nedosa A (2011) Dihydroartemisinin-Stimulated Hyperplasia of Rat Lung Smooth Muscles. Am J Appl Sci 9: 177-179.

20. Utoh-Nedosa UA, Kenneth OI Stanislaus NK (2011) Auto Negative-Feedback Effect is Responsible for the High Recrudescence Rate of Artemisinin Therapy and for Nontoxicity of Oral Dihydroartemisinin. Mol Pharm.

21. Hughes TA (1925) Effects of quinine on the sugar of the blood. Indian J Med Res 13: 2.

22. Rachael Rettner (2020) Mysterious Blood Clots in covid-19 Patients have Alarmed Medical Doctors.

23. Ahmed B, al-Howiriny TA, Mathew R (2002) Anti-hepatotoxic activity of Phylantus fraternus. Pharmazie 57: 855-856. [Crossref]

24. Campos AH, Schor N (1999) Phylantus niruri inhibits calcium oxalate endocytosis by renal tubular cells: its role in urolithiasis. Nephron 81: 393-397. [Crossref]
25. Freitas AM, Schor N, Boim MA (2002) The effect of Phylantus nirruri on urinary inhibitors of calcium oxalate crystallization and other factors associated with renal stone formation. BJU Int 89: 829-834. [Crossref]

26. Hukeri VI, Kalyani GA, Kakrani HK (1988) Hypoglycaemic activity of flavonoids of Phylantus nuriri in rats. Fitoterapia 59: 68-70.

27. Santos AR, De Campos RO, Miguel OG, Filho VC, Siani AC, et al (2000) Antinociceptive properties of extracts of new species of plants of genus Phylantus (Euphobiaceae). J Ethnopharmacol 72: 229-238. [Crossref]

28. Ramakrishhnan PN (1982) Oral hypoglycaemic effect of Phylantus niruri (Linn) leaves. Ind J Pharm Sci 44: 10-12.

29. Ueno H, Horie S, Nishi Y, Shogawa H, Kawasaki M, et al. (1988) Chemical and pharmaceutical studies on medicinal plants in Paraguay. Geranin, an angiotensinconverting enzyme inhibitor from 'paraparai mi', Phylantus niruri. J Nat Prod 51: 357359. [Crossref]

30. Srividya N, Periwal S (1995) Diuretic, hypotensive and hypoglycaemc effect of Phylantusamarus, J Indian Exp Biol 33: 861-864. [Crossref]

31. Wang MX, Cheng HW, Li YJ, Meng LM, Malik (1994) Observations of the efficacy of Phylantus spp in treating patients with chronic hepatitis B. Zhongguo Zhong Yao Za Zhi 19: 750-752.

32. Science and Technology Policy Research Institute (STEPRI), Council for Industrial Research (CSIR), Accra, Ghana, Ghana Herbal Pharmacopoeia, Kofi Busia (Ed), 2007.

33. Utoh-Nedosa UA, Akah PA, Okoli CO, Ojemudia TI, Nedosa IV, et al. (2011) Stimulatory Effects of Dihydroartemisinin on the Leucocyte Population of Wistar Albino Rats. Am J Infect Dis 7: 80-82.

34. Ogata T, Higuchi H, Mochida S, Matsumoto H, Kato A, et al. (1992). Hiv-1 reverse transcriptase inhibitor from Phylantus niruri. AIDS Res Hum Retroviruses 8: $1937-$ 1944. [Crossref]

35. Best TH, Smith RJ, Scott DA (1924) An insulin-like material in various tissues of the normal and diabetic animal. Amer J Physiol 68: 161-182.

36. Utoh-Nedosa UA, Mohammed S, Kenechi N, Goddy N, Onyedibe K, et al. (2012) Extra-Bone Marrow Sites of Haemopoesis: Dihydroartemisinin Effects in Wistar Albino Rats. Am J Appl Sci 9: 7-2.

37. Utoh-Nedosa UA (2014) Immune System and Body Defense Enhancement Effects of Oral Dihydroartemisinin in Wistar Albino Rats. J Pharm Pharmacol 2: 7.

38. Darvey TH, Crew (1972) A guide to human Parasitology for medical Practitioners, H.E Lewis Co Ltd, London.

Copyright: (C2020 Utoh-Nedosa UA. This is an open-access article distributed under the terms of the Creative Commons Attribution License, which permits unrestricted use, distribution, and reproduction in any medium, provided the original author and source are credited. 